\begin{tabular}{|c|l|}
\hline Title & On time analyticity of the Navier-Stokes equations in a rotating frame with spatially al most periodic data \\
\hline Author(s) & Giga, Y oshikazu; Jo, Hideaki; Mahalov, A lex; Y oneda, Tsuyoshi \\
\hline Citation & Hokkaido University Preprint Series in Mathematics, 860, 2-16 \\
\hline Issue Date & 2007 \\
\hline DOI & 10.14943/84010 \\
\hline Doc URL & http://hdl.handle.net/2115/69669 \\
\hline Type & bulletin (article) \\
\hline File Information & pre860.pdf \\
\hline
\end{tabular}

Instructions for use 


\title{
On time analyticity of the Navier-Stokes equations in a rotating frame with spatially almost periodic data
}

\author{
Yoshikazu Giga \\ Graduate School of Mathematical Sciences, University of Tokyo, 3-8-1 Komaba, \\ Meguro-ku, Tokyo 153-8914, Japan
}

Hideaki Jo

Graduate School of Mathematical Sciences, University of Tokyo, 3-8-1 Komaba, Meguro-ku, Tokyo 153-8914, Japan

Alex Mahalov

Department of Mathematics, Arizona State University, Tempe, AZ 85287-1804, USA

Tsuyoshi Yoneda

Graduate School of Mathematical Sciences, University of Tokyo, 3-8-1 Komaba, Meguro-ku, Tokyo 153-8914, Japan

\begin{abstract}
We consider the Navier-Stokes equations with the Coriolis force when intial data may not decrease at spatial infinity so that almost periodic data is allowed. We prove that the local-in-time solution is analytic in time when initial data is in $F M_{0}$, Fourier preimage of the space of all finite Radon measures with no point mass at the origin. When the initial data is almost periodic, this implies that the complex amplitude is analytic in time. In particular, a new mode cannot be created at any positive time.
\end{abstract}

Key words: Navier-Stokes equations, analyticity, frequency set, Coriolis force

Email addresses: labgiga@ms.u-tokyo.ac.jp (Yoshikazu Giga), hideaki@ms.u-tokyo.ac.jp (Hideaki Jo), mahalov@asu.edu (Alex Mahalov), yoneda@ms.u-tokyo.ac.jp (Tsuyoshi Yoneda). 


\section{Introduction and main results}

We consider the Cauchy problem for the three-dimensional Navier-Stokes equations with the Coriolis force:

$$
u_{t}+(u \cdot \nabla) u+\Omega e_{3} \times u-\Delta u=-\nabla p, \nabla \cdot u=0,\left.u\right|_{t=0}=u_{0},
$$

where $u=u(x, t)=\left(u_{1}(x, t), u_{2}(x, t), u_{3}(x, t)\right)$ is the unknown velocity vector field and $p=p(x, t)$ is the unknown scalar pressure at the point $x=$ $\left(x_{1}, x_{2}, x_{3}\right) \in \mathbf{R}^{3}$ in space and time $t>0$ while $u_{0}=u_{0}(x)$ is the given initial velocity field. Here $\Omega \in \mathbf{R}$ is the Coriolis parameter, which is twice the angular velocity of the rotation around the vertical unit vector $e_{3}=(0,0,1)$; the kinematic viscosity coefficient in normalized by one. By $\times$ we denote the exterior product, hence, the Coriolis term is represented by $e_{3} \times u=J u$ with the corresponding skew-symmetric $3 \times 3$ matrix $J$.

We would like to solve (1) when initial data $u_{0}$ is almost periodic, i.e., $u_{0}$ is formally of the form

$$
u_{0}(x)=\sum_{\lambda \in \Lambda} a_{\lambda} e^{i \lambda x}
$$

where $\Lambda$ (called a frequency set) is countable set in $\mathbf{R}^{3}$ and $a_{\lambda} \in \mathbf{C}^{3} \backslash\{0\}$. We would like to discuss time evolution of frequency sets. A naive space for this purpose is $B U C$, the space of all bounded uniformly continuous functions since almost periodic functions in the sense of Bohr belong to $B U C$. The problem (1) is well-posed when $\Omega=0$ as proved in [10]. However, when $\Omega \neq 0$ it is shown in [11] that the linearized problem around $u=0$ is ill-posed in the space $B U C$. Sawada [20] and Hieber and Sawada [15] proved unique local existence for initial data in a homogeneous Besov space $\dot{B}_{\infty, 1}^{0}$, which is strictly smaller than $B U C$. The estimate for the existence time is improved by [11]. However, non of these results gives a uniform estimate for the existence time in the speed of rotation $\Omega \in \mathbf{R}$, the precondition to consider fast singular oscillating limits $\Omega \rightarrow \pm \infty$ and prove global existence for large fixed $\Omega$.

To overcome this difficulty the space $F M_{0}$ (Fourier preimage of the space of all finite Radon measures with no point mass at the origin) is introduced in [12]. This space $F M_{0}$ is strictly smaller than $\dot{B}_{\infty, 1}^{0}$ as proved in [12]. However, the existence time can be taken uniformly in $\Omega$ as proved in [12].

The global existence of a regular solution of (1) for large $|\Omega|$ were obtained in $L^{2}$-setting for the periodic domains such as cylinder with infinite length in the direction of the rotating axis (periodic or zero stress boundary conditions in the vertical direction) or spatial lattices [4], [5], [17]. In this regard, for the Euler equations, Nicolaenko, Bardos Golse and the third author [18] proved uniform local existence and long-time regularity for initial data in $H^{4}$. 
In $F M_{0}$ setting a global solvability is proved for small Reynolds number but all $\Omega$ under some conditions on frequency set of $u_{0}$ [13].

Our goal in this paper is to prove the analyticity of the solution $u$ in time. Here is a typical result.

Theorem 1.1. Assume that $u_{0} \in F M_{0}$ with $\operatorname{div} u_{0}=0$. Let $u$ be the unique mild solution $u=u(t) \in C\left([0, T], F M_{0}\right)$ of (1) (see [12]). Then $u$ is analytic in $(0, T)$ in the sense it can be extended to be a holomorphic function with values in $F M_{0}$ in a complex neighborhood of $(0, T)$.

For the case $\Omega=0$ we may state in the framework of $B U C$.

Theorem 1.2. Assume that $\Omega=0$ and $u_{0} \in B U C$ with $\operatorname{div} u_{0}=0$. Let $u$ be the unique mild solution $u=u(t) \in C([0, T], B U C)$ of (1). Then $u$ is analytic in $(0, T)$ with values in $B U C$.

The analyticity in time for the Navier-Stokes equations has been discussed by many authors in various settings. The first result in this direction was established by Masuda[19]. He proved that if $u \in C\left([0, T] ; H^{2}(D)\right)$ is a weak solution of (1) with $\Omega=0$, then $u$ is analytic in $(0, T)$ with value in $H^{2}(D)$. In [19] $D$ is assumed to be a bounded domain and the Dirichlet condition is imposed. Also, an analytic exterior force is allowed. (His result works for unbounded domains in $L^{2}$-setting too.) This result is extended by the first author [9] for $L^{p}$-setting. Foias and Temam[8] and Iooss[16] also discuss the time analyticity when $\Omega=0$ in a different setting. However, non of these results apply to the space containing nondecaying functions such as almost periodic functions.

We now consider an almost periodic initial data $u_{0} \in F M_{0}$ of the form (2). As pointed out in [12] the solution $u(t) \in F M_{0}$ is almost periodic as proved in [14] for other spaces. It is interesting to study evolution of the frequency set.

Theorem 1.3. Let $u$ be the mild solution of (1) in $[0, T]$ with initial data $u_{0} \in F M_{0}$ which is almost periodic of the form (2). Then the frequency set $\Lambda(u(\cdot, t))$ of $u(\cdot, t)$ is contained in the additive free semigroup $G$ generated by $\Lambda$. Thus $u$ is of the form

$$
u(x, t)=\sum_{\lambda \in G} a_{\lambda}(t) e^{i \lambda \cdot x}
$$

where the amplitude $a_{\lambda}(t) \in \mathbf{C}^{3}$ and satifies

$$
\sup _{0<t<T} \sum_{\lambda \in G}\left|a_{\lambda}(t)\right|<\infty .
$$


(The sum converges in absolute sense uniformly in $x \in \mathbf{R}^{3}$.)

Since the amplitude function $a_{\lambda}(t)$ can be calculated by

$$
a_{\lambda}(t)=\mathrm{M}\left[u e^{-i \lambda \cdot x}\right]
$$

where $\mathrm{M}[f]=\lim _{R \rightarrow \infty}\left|C_{R}\right|^{-1} \int_{C_{R}} f d x$ is the spatial averaging operator; $C_{R}$ denote the cube $\left\{x=\left(x_{1}, x_{2}, x_{3}\right)|| x_{i} \mid \leq R, 1 \leq i \leq 3\right\}$; see [7]. By the time analyticity result we are able to prove the analyticity of the amplitude function.

Theorem 1.4. Assume the same hypotheses of either Theorem 1.1 or 1.2. Then the complex amplitude function $a_{\lambda}(t)$ is analytic in time $t>0$.

This implies that there is no sudden creation of new mode at $t_{0}>0$ or there is no chance that some mode vanishes identically from some $t_{0}>0$. All modes appearing for $t>0$ are created instantaneously. There is no waiting time for exciting amplitude dynamics corresponding to any frequency.

The work of the first author was partly supported by the Grant-in-Aid for Scientific Research, No. 18204011, No. 17654037, the Japan Society of the Promotion of Science (JSPS). He was also partly supported by the Grant-inAid for formation of COE 'Mathmatics of Nonlinear Structures via Singularities' (Hokkaido University) sponsored by JSPS. The work of the third author was partly supported by AFOSR contract FA9550-05-1-0047. The work of the fourth author was partly supported by the Grant-in-Aid for JSPS Fellows.

\section{Persistence of almost periodicity and frequency set}

We begin by recalling the notion of almost periodicity in the sense of Bohr [1], [7]. Let $f$ be in $B U C\left(\mathbf{R}^{n}\right)$. We say $f$ is almost periodic if the set

$$
\Sigma_{f}:=\left\{f(\cdot+\xi) \mid \xi \in \mathbf{R}^{n}\right\} \subset L^{\infty}\left(\mathbf{R}^{n}\right)
$$

is relatively compact in $L^{\infty}\left(\mathbf{R}^{n}\right)$. (Uniform continuity is redundant. In fact, if $f$ must be uniformly continuous [1],[7].) If $f$ is periodic in $x$, clearly, $f$ is almost periodic since $\Sigma_{f}$ is a torus.

We next recall the fact that if $u_{0}$ is almost periodic so is the solution $u(\cdot, t)$ of (1). To state more precisely we recall an integral equation which is formally equivalent to (1):

$$
u(t)=e^{-A(\Omega) t} u_{0}-\int_{0}^{t} \operatorname{div} e^{-A(\Omega)(t-s)} \mathbf{P}(u(s) \otimes u(s)) d s,
$$


where $A(\Omega)=-\Delta+\Omega \mathbf{S}, \mathbf{S}=\mathbf{P} J \mathbf{P}$ (Poincaré-Riesz operator) and $\mathbf{P}=(I+$ $\left.R_{i} R_{j}\right)_{i \leq i, j \leq 3}$ (Helmholtz projection); here, $R_{j}$ denotes the Riesz operator $R_{j}=$ $(-\Delta)^{-1 / 2} \partial / \partial x_{j}$. Instead of considering solutions of (1), we rather consider a solution of (4) in $C\left([0, T], F M_{0}\right)$ (or $C([0, T], B U C)$ for $\Omega=0$ ) which is called a mild solution. The relation of (1) and (2) is discussed in [12] and [10]; we do not repeat it here.

\section{Theorem 2.1.}

(i) Assume that $u_{0} \in F M_{0}$ with div $u_{0}=0$ is almost periodic. Let $u \in$ $C\left([0, T], F M_{0}\right)$ be the unique mild solution of $(4)$. Then $u(\cdot, t)$ is almost periodic in $\mathbf{R}^{3}$ for $t \in[0, T]$.

(ii) Assume that $\Omega=0$. Assume that $u_{0} \in B U C\left(\mathbf{R}^{3}\right)$ with $\operatorname{div} u_{0}=0$ is almost periodic. Let $u \in C([0, T], B U C)$ be the unique mild solution of (4). Then $u(\cdot, t)$ is almost periodic in $\mathbf{R}^{3}$ for $t \in[0, T]$.

The second statement (ii) is found in [14] (Theorem 2.3). The first statement (i) is not explicitly written but the proof parallels that of (ii). We just give a sketch of the proof of (i) for the reader's convenience.

Sketch of the proof of (i). For the solution $u(t)=u(\cdot, t)$ let $S(t)$ denote the mapping $u_{0} \mapsto u(t)$.

Since (1) is invariant under translation in the space variables, the solution $u_{\eta}$ with initial data $u_{0 \eta}(x)=u_{0}(x+\eta), \eta \in \mathbf{R}^{3}$ fulfills $u_{\eta}(x, t)=u(x+\eta, t)$. Thus $S(t)$ maps $\Sigma_{u_{0}}$ onto $\Sigma_{u(t)}$. We notice that

$$
\sup _{0<t<T}\left\|u_{\eta}\right\|_{F M}(t)=\sup _{0<t<T}\|u\|_{F M}(t) .
$$

We now apply a uniform continuity dependence on initial data described below in Lemma 2.2 and observe that $S(t)$ is a well-defined continuous mapping from the closure $\bar{\Sigma}_{u_{0}}$ to $\bar{\Sigma}_{u(\cdot, t)}$ in $F M_{0}$. Thus if $\bar{\Sigma}_{u_{0}}$ is compact in $F M_{0}$, so is $\bar{\Sigma}_{u(\cdot, t)}$. One may replace $F M_{0}$ by $B U C$ as proved below in Lemma 2.4. The proof is now complete.

Lemma 2.2.(Continuity with respect to initial data.) For a given $N$ and $T$ let $B(N)$ denote the set of all $u_{0} \in F M_{0}$ such that

$$
\sup _{0<t<T}\left\|S(t) u_{0}\right\|_{F M} \leq N .
$$

Then $S(t)$ is a Lipschitz continuous on $B(N)$ to $F M_{0}$ with a constant depending only on $N$ and $T$.

The proof parallels that of [14] ( Proposition 3.2). It is essentially contained in [12] (Section 3). 
We conclude this section by proving Theorem 1.3. For this purpose we clarify the meaning of (2) when $u_{0} \in F M$.

Lemma 2.3. Assume that $f$ is almost periodic and belongs to $F M\left(\mathbf{R}^{n}\right)$. Then $f$ is a Fourier sum of the form

$$
f(x)=\sum_{\lambda \in \Lambda} a_{\lambda} e^{i \lambda x} \quad \text { with } \quad \sum_{\lambda \in \Lambda}\left|a_{\lambda}\right|<\infty,
$$

where $a_{\lambda} \in \mathbf{C}^{n}$ and $\Lambda$ is a countable subset of $\mathbf{R}^{n}$. Conversely, if $f$ has the form (5), then $f \in F M\left(\mathbf{R}^{n}\right)$ and $f$ is almost periodic.

Proof. Let $\mu$ be the Fourier image $\hat{f}$ of $f$. We split $\mu \in M$ as

$$
\mu=\mu_{p}+\mu_{c}
$$

where $\mu_{p}$ is the point mass part and $\mu_{c}$ is the continuous part. The inverse Fourier image $F^{-1} \mu_{p}$ of $\mu_{p}$ is a Fourier sum as in (5). So $F^{-1} \mu_{p}$ is almost periodic [7]. We should prove that $f_{c}=F^{-1} \mu_{c}$ is zero if it is almost periodic. As in [7] it suffices to prove that all Fourier coefficients (complex amplitudes) of $f_{c}$ must be zero which is obtained by calculating the average

$$
\mathrm{M}\left[f_{c} e^{-i \lambda \cdot x}\right]=\lim _{R \rightarrow \infty}(2 R)^{-n} \int_{C_{R}} f_{c} e^{-i \lambda \cdot x} d x .
$$

We observe that

$$
\begin{aligned}
& (2 \pi)^{n} \int_{C_{R}} f_{c} e^{-i \lambda \cdot x} d x=\int_{\mathbf{R}^{n}} \int_{C_{R}} e^{i x \cdot \xi} e^{-i \lambda \cdot x} \mu_{c}(d \xi) d x=\int_{\mathbf{R}^{n}} \int_{C_{R}} e^{i x \cdot \eta} \mu_{c}^{\lambda}(d \eta) d x \\
& =R^{n} \int_{\mathbf{R}^{n}}\left(\int_{C_{1}} e^{i y \cdot R \eta} d y\right) \mu_{c}^{\lambda}(d \eta),
\end{aligned}
$$

where $\mu_{c}^{\lambda}$ is the translation of $\mu_{c}$ defined by $\mu_{c}^{\lambda}(B)=\mu_{c}(B+\{\lambda\})$ for Borel set $B$. Since

$$
\lim _{R \rightarrow \infty} \int_{C_{1}} e^{i y \cdot R \eta} d y=0 \text { for all } \eta \neq 0
$$

and $\mu_{c}^{\lambda}$ has no point mass at the origin, by the Lebesgue dominant convergence theorem we conclude that $\mathrm{M}\left[f_{c} e^{-i \lambda \cdot x}\right]=0$ for all $\lambda \in \mathbf{R}^{n}$.

The converse is clear since $\sum\left|a_{\lambda}\right|=\|f\|_{F M}$ and the uniform limit of the Fourier sum is almost periodic.

Lemma 2.4. A function $f \in F M_{0}\left(\mathbf{R}^{n}\right)$ is almost periodic if and only if $\Sigma_{f}$ is relatively compact in $F M_{0}\left(\mathbf{R}^{n}\right)$. 
Proof. It suffices to prove 'only if' part since $F M_{0} \subset L^{\infty}$ is continuous. We may assume that $f$ is a scalar valued function. Suppose that $\Sigma_{f}\left(\subset F M_{0}\right)$ is relatively compact in $L^{\infty}\left(\mathbf{R}^{n}\right)$. Then any sequence $\left\{f_{n_{k}}\right\} \subset \Sigma_{f}$ has a convergent subsequence $\left\{f_{\ell}=f\left(\cdot+\eta_{k(\ell)}\right)\right\} \rightarrow f_{0}$ in $L^{\infty}\left(\mathbf{R}^{n}\right)$ as $\ell \rightarrow \infty$. It suffices to prove that $f_{\ell} \rightarrow f_{0}$ in $F M_{0}$. By (5) we have $f_{0}(x)=\sum_{\lambda \in \Lambda} a_{\lambda} e^{i \lambda x}$ and $f_{\ell}(x)=\sum_{\lambda \in \Lambda} a_{\lambda} e_{\lambda \ell} e^{i \lambda x}$, where $e_{\lambda \ell}:=\exp \left(i \lambda n_{k(\ell)}\right)$. Since $f_{\ell} \rightarrow f_{0}$ in $L^{\infty}\left(\mathbf{R}^{n}\right)$, we have

$$
\lim _{\ell \rightarrow \infty} \mathrm{M}\left[\left(f_{\ell}-f_{0}\right) e^{-i \mu \cdot x}\right]=0 \quad \text { for all } \quad \mu \in \Lambda .
$$

In other words

$$
a_{\mu} e_{\mu \ell} \rightarrow a_{\mu} \quad \text { as } \quad \ell \rightarrow \infty
$$

for all $\mu \in \Lambda$. Thus we conclude that

$$
\left\|f_{\ell}-f_{0}\right\|_{F M}=\sum_{\lambda \in \Lambda}\left|a_{\lambda}-a_{\lambda} e_{\lambda \ell}\right| \rightarrow 0
$$

since $\sum_{\lambda \in \Lambda}\left|a_{\lambda}\right|=\sum_{\lambda \in \Lambda}\left|a_{\lambda} e_{\lambda \ell}\right|<\infty$. (The same statement holds even if we replace $F M_{0}$ by $F M$.)

Proof of Theorem 1.3. Assume that $f, g \in F M$ are almost periodic. Let $\Lambda(f)$ denote the frequency set, i.e., it is the set of all $\lambda \in \Lambda$ such that $a_{\lambda} \neq 0$. Then by Lemma 2.3 it is easy to see that

$$
\Lambda(f g) \subset \Lambda(f)+\Lambda(g)=\{a+b \mid a \in \Lambda(f), b \in \Lambda(g)\} .
$$

Since the solution of (4) is the limit of the successive approximation

$$
\begin{aligned}
u_{m+1}(t) & =e^{-A(\Omega) t} u_{0}-\int_{0}^{t} \operatorname{div} e^{-A(\Omega)(t-s)} \mathbf{P}\left(u_{m}(s) \otimes u_{m}(s)\right) d s(m \geq 1), \\
u_{1}(t) & =e^{-A(\Omega) t} u_{0}
\end{aligned}
$$

and $e^{-A(\Omega) t}, \operatorname{div} e^{-A(\Omega) t} \mathbf{P}$ does not grow the frequency set, we observe that $u_{m}(t)$ is a Fourier sum of the form (5) and that

$$
\begin{aligned}
& \Lambda\left(u_{1}(t)\right) \subset \Lambda\left(u_{0}\right), \\
& \Lambda\left(u_{m+1}(t)\right) \subset \bigcup_{0 \leq \tau<t} \Lambda\left(u_{m}(\tau)\right)+\Lambda\left(u_{m}(\tau)\right), m=1,2 \cdots .
\end{aligned}
$$

This implies that $\Lambda\left(u_{m}(t)\right) \subset G$, where $G$ is the free additive semigroup generated by $\Lambda$. Since the convergence of $u_{m}$ in $C\left(\left[0, T_{0}\right], F M_{0}\right)$ to the solution $u$ for small $T_{0}$ has been established in [12] (Section 3), and since $\sum_{\lambda \in G}\left|a_{\lambda}(t)\right|=\|u(t)\|_{F M}$, we obtain (3) in $\left(0, T_{0}\right]$. We repeat this argument starting form $t=T_{0}$ with $\Lambda=G$ and obtain (1.3) in $\left[T_{0}, T_{0}+T_{1}\right]$ with $T_{1}>0$. 
We repeat this argument finitely many times to conclude (3) in $[0, T]$ since the amount of increment of time interval in each step can be taken uniformly since it depends only on the size of initial data in $F M$.

\section{Remark 2.5.}

(i) Our terminology is slightly different from [13], where it gives an impression that frequency set is the support of $\hat{f}$, which is the closure of our frequency set of almost periodic function $f$.

(ii) The assertion that the frequency set of $u$ is contained in $G$ is still valid in $B U C$ setting when $\Omega=0$ since we have $\Lambda(f g) \subset \Lambda(f)+\Lambda(g)$ for almost periodic $f, g \in B U C\left(\mathbf{R}^{n}\right)$; note that an almost priodic function can be approximated by trigonometric polynomials in uniform sense.

\section{Time analyticity}

We shall prove Theorem 1.1 and 1.2. Theorem 1.4 is an easy collorary of Theorem 1.1 and 1.2.

We recall the definition of holomorphic functions with values in a (complex) Banach space $X$. Let $D$ be an open set in $\mathbf{C}$. We say that a function from $D$ to $X$ is (strongly) holomorphic (with values in $X$ ) in $D$ if $f$ is strongly differentiable at every point of $D$, i.e., for each $z \in D$ there exists an $L \in X$ such that

$$
\lim _{h \rightarrow 0, h \in \mathbf{C}} \frac{1}{|h|}\|f(z+h)-f(z)-h L\|_{X}=0 .
$$

For basic properties of Banach valued holomorphic functions the reader is referred to classical books by Yosida [22] and by Berger [3]. Let $H^{\infty}(D, X)$ be the space of all bounded holomorphic functions in $D$ with values in $X$. It is a Banach space equipped with the supremun norm defined by

$$
\|f\|_{H^{\infty}(D)}=\sup \left\{\|f(z)\|_{X}: z \in D\right\} .
$$

We still use such notation when $D$ is an open set in $\mathbf{C}^{2}$; in this case we say that $f: D \rightarrow X$ is holomorphic if $f=f(t, s)$ is holomorphic in each variable $t$ and $s$. For $\sigma \in(0, \pi / 2)$ and $T>0$ we set

$$
\begin{aligned}
& S(T, \sigma)=\{t \in \mathbf{C}:|\arg t|<\sigma, \operatorname{Re} t<T\} \\
& Z(T, \sigma)=\{(t, s) \in S(T, \sigma) \times S(T, \sigma): \operatorname{Re}(t-s)>0\} \\
& Z_{\epsilon}(T, \sigma)=\{(t, s) \in Z(T, \sigma): \operatorname{Re}(t-s)>\varepsilon\} \text { for } \varepsilon>0,
\end{aligned}
$$


where Re $z$ denotes the real part of a complex number $z$. The next Theorem is elementary but a key for our argument. It is implicitly used in the proof of [9] (Lemma 2).

Theorem 3.1. Assume that $f \in H^{\infty}\left(Z_{\varepsilon}(T, \sigma), X\right)$ for each $\varepsilon \in(0, T)$. Assume that there is an positive integrable functions $\varphi$ on $(0, T)$ such that

$$
\|f(t, s)\|_{X} \leq \varphi(\operatorname{Re}(t-s)) \text { for }(t, s) \in Z(T, \sigma) .
$$

Then $F(t):=\int_{0}^{t} f(t, s) d s$ is in $H^{\infty}(S(T, \sigma), X)$ with

$$
\|F\|_{H^{\infty}(S(T, \sigma))} \leq \int_{0}^{T} \varphi(\tau) d \tau
$$

Here, we interpret that the complex integration is made along the segment connecting two ends 0 and $t$.

Proof. We set

$$
F_{\delta}(t)=\int_{0}^{t(1-\delta)} f(t, s) d s
$$

for $\delta \in(0,1)$. It suffices to prove that $F_{\delta} \in H^{\infty}(S(T, \sigma), X)$ since $F$ is a uniform limit of $F_{\delta}$ as $\delta \rightarrow 0$ by the assumption (6); the estimate (7) easily follows from (6).

Since the boundedness of $F_{\delta}$ in $S(T, \sigma)$ is clear, it suffices to prove that $F_{\delta}$ is holomorphic in $S(T, \sigma)$. For $t \in S(T, \sigma)$ we take $h \in \mathbf{C}$ small so that $t+h \in S(T, \sigma)$. Applying Cauchy's integration theorem to change the path we observe that

$$
\begin{aligned}
F_{\delta}(t+h)-F_{\delta}(t) & =\int_{0}^{\alpha(t+h)}(f(t+h, s)-f(t, s)) d s \\
& +\int_{0}^{\alpha(t+h)} f(t, s) d s-\int_{0}^{\alpha t} f(t, s) d s \\
& =\int_{0}^{\alpha(t+h)}(f(t+h, s)-f(t, s)) d s+\int_{\alpha t}^{\alpha(t+h)} f(t, s) d s \\
& =: J_{1}+J_{2}
\end{aligned}
$$

with $\alpha=1-\delta$. We recall a form of Taylor's expansion (See [2]):

$$
g(z)=\sum_{k=0}^{n-1} \frac{g^{(k)}(a)}{k !}(z-a)^{k}+\left(\frac{1}{2 \pi i} \int_{C} \frac{g(\zeta)}{(\zeta-a)^{n}(\zeta-z)} d \zeta\right) \cdot(z-a)^{n} .
$$


Here $g$ is an holomorphic function defined in a domain $D \subset \mathbf{C}$ and $a \in D ; C(\subset$ $D)$ is a circle (oriented counterclockwise) centered at $a$ which encloses $z$.

We use (8) with $n=2, a=t, z=t+h, g(z)=f(z, s)$. We fix the circle $C:|\zeta-t|=r$ so that $r<\operatorname{dist}(t, \partial S(T, \sigma))$. We take $h$ small so that $|h|<r$. Then

$$
\frac{J_{1}}{h}-\int_{0}^{\alpha(t+h)} \frac{\partial f}{\partial t}(t, s) d s=h \int_{0}^{\alpha(t+h)}\left\{\frac{1}{2 \pi i} \int_{C} \frac{f(\zeta, s)}{(\zeta-(t+h))(\zeta-t)^{2}} d \zeta\right\} d s
$$

The norm of the right hand side in $X$ is dominated by

$$
\frac{h}{2 \pi} \frac{M_{\varepsilon}}{(r-|h|) r^{2}} \cdot 2 \pi r \text { with } M_{\varepsilon}=\|f\|_{H^{\infty}\left(Z_{\varepsilon}(T, \sigma)\right)} .
$$

Similarly, we apply (8) with $n=1, a=\alpha t, z=s$ and $g(z)=f(t, z)$ to observe that

$$
\frac{J_{2}}{h}-\alpha f(t, \alpha t)=\int_{\alpha t}^{\alpha(t+h)}\left\{\frac{1}{2 \pi i} \int_{C} \frac{f(\zeta, s)}{(\zeta-\alpha t)(\zeta-s)} d \zeta \cdot(s-\alpha t)\right\} d s .
$$

Here we fix the critical $C:|\zeta-\alpha t|=r$ so that $r<\operatorname{dist}(\alpha t, \partial S(T, \sigma))$ and take $h$ small so that $\alpha|h|<r$. The norm of the right hand side is dominated by

$$
\frac{1}{2 \pi} \frac{M_{\varepsilon}}{r(r-\alpha|h|)} 2 \pi r \cdot \alpha|h|
$$

Thus we conclude that

$$
\lim _{|h| \rightarrow 0} \frac{1}{|h|}\left\|F_{\delta}(t+h)-F_{\delta}(t)-h\left\{\int_{0}^{\alpha t} \frac{\partial f}{\partial t}(t, s) d s+\alpha f(t, \alpha t)\right\}\right\|_{X}=0,
$$

that is to say that $F_{\delta}$ is (complex) differentiable at $t \in S(T, \sigma)$.

\section{Lemma 3.2}

If $u \in H^{\infty}(S(T, \sigma) ; F M)$, then

$$
f(t, s):=\operatorname{div} e^{-(t-s) A(\Omega)} \mathbf{P} u(s) \otimes u(s)
$$

belongs to $H^{\infty}\left(Z_{\varepsilon}(T, \sigma) ; F M\right)$ for all $\varepsilon \in(0, T)$. Moreover,

$$
\|f(t, s)\|_{F M} \leq \frac{C}{(\operatorname{Re}(t-s))^{\frac{1}{2}}}\|u\|_{F M}^{2}
$$

holds with constant $C$ independent of $t, s$ and $u$.

Proof. First we show that the function $f(t, s)$ belongs to $H^{\infty}\left(Z_{\varepsilon}(T, \sigma) ; F M\right)$ if $u \in H^{\infty}(S(T, \sigma) ; F M)$ briefly. Let $\sigma^{*}(A)$ be the symbol of the operator $A$. 
We shall recall the symbol of operators appearing in $\operatorname{div} e^{-(t-s) A(\Omega)} \mathbf{P}$. As in [11] the symbol of $e^{t \Omega \mathbf{S}}$ is given by

$$
\sigma^{*}\left(e^{t \Omega \mathbf{S}}\right)=\exp \left(\Omega t \frac{\xi_{3}}{|\xi|} \mathbf{R}(\xi)\right)
$$

where $\mathbf{R}(\xi)$ is the symbol of the vector Riesz operator, i.e.,

$$
\mathbf{R}(\xi)=\frac{1}{|\xi|}\left(\begin{array}{ccc}
0 & -\xi_{3} & \xi_{2} \\
\xi_{3} & 0 & -\xi_{1} \\
-\xi_{2} & \xi_{1} & 0
\end{array}\right)
$$

The symbol of $e^{-t A(\Omega)}$ and $\mathbf{P}$ equal to

$$
\sigma^{*}\left(e^{-A(\Omega) t}\right)=e^{-|\xi|^{2} t} \sigma^{*}\left(e^{-t \Omega \mathbf{S}}\right) \quad \text { and } \quad \sigma^{*}(\mathbf{P})=\left(\delta_{j k}-\frac{\xi_{j} \xi_{k}}{|\xi|^{2}}\right)
$$

Thus the symbol of dive $e^{-(t-s) A(\Omega)} \mathbf{P}$ is holomophic in $(t, s)$ from $Z(T, \sigma) \rightarrow$ $B U C\left(\mathbf{R}^{3}\right)$, since the symbol $\sigma^{*}\left(\operatorname{div} e^{-t A(\Omega)} \mathbf{P}\right)$ can be extended to be continuous up to the origin. Moreover, $\sigma^{*}\left(\operatorname{div} e^{-(t-s) A(\Omega)} \mathbf{P}\right) \in H^{\infty}\left(Z_{\varepsilon}(T, \sigma), B U C\left(\mathbf{R}^{3}\right)\right)$. Thus for $\mu \in M$, the associate measure $\mu\left\lfloor\sigma^{*}\left(\operatorname{div} e^{-(t-s) A(\Omega)} \mathbf{P}\right)\right.$ is in $H^{\infty}\left(Z_{\varepsilon}(T, \sigma), M\right)$. We now conclude that $f \in H^{\infty}\left(Z_{\varepsilon}(T, \sigma), F M\right)$.

Next we show (10). Note that $\|\mathbf{P} u\|_{F M} \leq\|u\|_{F M}$ holds (see [12]). By [12] we have

$$
\left\|e^{-t \Omega \mathbf{S}}\right\|_{F M \rightarrow F M} \leq \sup _{\xi \in \mathbf{R}^{3}}\left|\sigma^{*}\left(e^{-t \Omega \mathbf{S}}\right)\right|,
$$

where $|\cdot|$ denotes the operator norm of $3 \times 3$ matrix from the Euclidean space $\mathbf{R}^{3}$ into itself. Note that the symbol of $e^{-t \Omega \mathbf{S}}$ is expressed in (11) and it is unitary in real $t$. There exists a constant $C^{*}$ depending only on $\Omega, \sigma$ and $T$ satisfying

$$
\left\|e^{-(t-s) \Omega \mathbf{S}}\right\|_{F M \rightarrow F M} \leq \sup _{\xi \in \mathbf{R}^{3}}\left|\sigma^{*}\left(e^{-\operatorname{Im}(t-s) \Omega \mathbf{S}}\right)\right| \leq C^{*}
$$

for all $(t, s) \in Z(\sigma, T)$, where $\operatorname{Im} z$ denotes the imaginary part of a complex number $z$.

Thus we have

$$
\begin{aligned}
\|f(t, s)\|_{F M} & \leq\left\|\operatorname{div} e^{(t-s) \Delta}\right\|_{F M \rightarrow F M}\|\mathbf{P}\|_{F M \rightarrow F M}\left\|e^{-(t-s) \Omega \mathbf{S}}\left(u_{m} \otimes u_{m}\right)\right\|_{F M} \\
& \leq C^{*}\left\|\operatorname{div} e^{(t-s) \Delta}\right\|_{F M \rightarrow F M}\left\|\left(u_{m} \otimes u_{m}\right)\right\|_{F M} .
\end{aligned}
$$

Since $F\left[\partial_{x_{j}} e^{t \Delta} g\right]=\hat{g} \cdot i \xi_{j} e^{-t|\xi|^{2}}$ for $g \in F M$, we have 


$$
\begin{aligned}
\left\|\partial_{x_{j}} e^{t \Delta} g\right\|_{F M} & =\left\|i \xi_{j} e^{-t|\xi|^{2}}\right\|_{L^{\infty}}\|g\|_{F M} \\
& \leq \sup _{|\xi| \geq 0}|\xi| e^{-\operatorname{Re} t|\xi|^{2}} \mid\|g\|_{F M} \leq \frac{1}{(2(\operatorname{Re} t) e)^{\frac{1}{2}}}\|g\|_{F M}
\end{aligned}
$$

(cf.[12]). Therefore we obtain

$$
\|f(t, s)\|_{F M} \leq \frac{C^{*}}{(2(\operatorname{Re}(t-s)) e)^{\frac{1}{2}}}\left\|u_{m} \otimes u_{m}\right\|_{F M} \leq \frac{C^{*}}{(2(\operatorname{Re}(t-s)) e)^{\frac{1}{2}}}\left\|u_{m}\right\|_{F M}^{2} .
$$

Thus, we have the desired estimate with $C=\frac{C^{*}}{\sqrt{2 e}}$.

\section{Proof of Theorem 1.1.}

We use successive approximation method. Let $\left\{u_{m}\right\}_{m \in \mathbb{N}}$ be as follows ;

$$
\begin{aligned}
u_{1}(t) & =e^{-t A(\Omega)} u_{0}, \\
u_{m+1}(t) & =u_{1}(t)-\int_{0}^{t} e^{-(t-s) A(\Omega)} \mathbf{P} \operatorname{div}\left(u_{m} \otimes u_{m}\right)(s) d s .
\end{aligned}
$$

First, we prove $u_{m} \in H^{\infty}\left(S(T, \sigma), F M_{0}\right)$ by induction on $m$.

Since $e^{-t A(\Omega)}$ is an analytic semigroup, $u_{1} \in H^{\infty}\left(S(T, \sigma), F M_{0}\right)$. Assume that $u_{m} \in H^{\infty}\left(S(T, \sigma), F M_{0}\right)$, then it follows immediately from Theorem 3.1 and Lemma 3.2 that $u_{m+1} \in H^{\infty}\left(S(T, \sigma), F M_{0}\right)$.

Next, we prove $\left\{u_{m}\right\}_{m \in \mathbb{N}}$ becomes a Cauchy sequence for sufficiently small $T_{0}$. We now define

$$
K_{m}\left(T_{0}\right)=\sup _{s \in S\left(T_{0}, \sigma\right)}\left\|u_{m}(s)\right\|_{F M}, L_{m}\left(T_{0}\right)=\sup _{s \in S\left(T_{0}, \sigma\right)}\left\|u_{m}(s)-u_{m-1}(s)\right\|_{F M} .
$$

Applying the above estimates, we have

$$
K_{m+1}\left(T_{0}\right) \leq C^{*}\left\|u_{0}\right\|_{F M}+\left|\int_{0}^{t} \frac{C}{(\operatorname{Re}(t-s))^{\frac{1}{2}}}\left\|u_{m}\right\|_{F M}^{2} d s\right| .
$$

Since

$$
\left|\int_{0}^{t} \frac{d s}{(\operatorname{Re}(t-s))^{\frac{1}{2}}}\right|=\frac{2|t|}{(\operatorname{Re} t)^{\frac{1}{2}}}<\frac{2 T_{0}^{\frac{1}{2}}}{\cos \sigma}
$$

we obtain $K_{m+1}\left(T_{0}\right) \leq C^{*}\left\|u_{0}\right\|_{F M}+C^{\prime} K_{m}^{2}$, where $C^{\prime}=\frac{2 T_{0}^{\frac{1}{2}}}{\cos \sigma} C^{*}$. Now, we take $T_{0}$ so small that both $C^{*} \leq \frac{3}{2}$ and $C^{\prime} \leq \frac{1}{6\left\|u_{0}\right\|_{F M}}$ holds. Under these assumptions, we have

$$
K_{m+1}\left(T_{0}\right) \leq \frac{3}{2}\left\|u_{0}\right\|_{F M}+\frac{\left(K_{m}\left(T_{0}\right)\right)^{2}}{6\left\|u_{0}\right\|_{F M}}
$$


and one can easily check that $K_{m}\left(T_{0}\right) \leq 3\left\|u_{0}\right\|_{F M}$ holds for all $m \in \mathbb{N}$.

Next, we estimate $\left\|u_{m+1}-u_{m}\right\|_{F M}$. By the similar argument together with the uniform boundedness, we obtain

$$
L_{m+1}\left(T_{0}\right) \leq \frac{36 T_{0}^{\frac{1}{2}} C^{*}}{\cos \sigma}\left\|u_{0}\right\|_{F M} L_{m}\left(T_{0}\right) .
$$

It follows that if we take $T_{0}$ so small that $\frac{36 T_{0}^{\frac{1}{2}} C^{*}}{\cos \sigma}\left\|u_{0}\right\|_{F M}<1$, then $\left\{u_{m}\right\}_{m \in \mathbb{N}}$ becomes a Cauchy sequence in $H^{\infty}\left(S\left(T_{0}, \sigma\right), F M_{0}\right)$.

Since the solution in $\left[0, T_{0}\right]$ is unique, this implies that $u$ is analytic with values in $F M_{0}$. Since $u(s) \in F M_{0}$ for all $s \in(0, T)$, starting from $u(s)$ and conclude that $u$ is analytic in some open interval $\left(s, s+T_{0}\right)$. Since $u$ can be taken anbitrary, this implies that $u$ is analytic in $(0, T)$. (Note that we need not to assume that $u$ is continuous up to $t=0$ and $t=T$. We may replace $[0, T]$ by $(0, T)$ to get analyticity in $(0, T)$. )

\section{Proof of Theorem 1.2}

It suffices to prove that the statement of Lemma 3.2 still holds even if we replace $F M$ by $B U C$ provided that we assume $\Omega=0$. (The semigruop $e^{t \mathbf{S}}$ is not bounded in $B U C$.) The analyticity of $f(t, s)$ easily follows from the analyticity of the semigroup $e^{t \Delta}$ in $B U C$. To prove (10) we need an estimate for $\nabla e^{t \Delta} \mathbf{P}$ which is proved in the Appendix. The remaining proof is the same as that of Theorem 1.1.

\section{Appendix.}

We give a short direct proof of the key estimate for the derivative of the heat semigroup in the proof of Theorem 1.2.

Lemma A. There is a universal constant $C$ such that

$$
\left\|\nabla e^{t \Delta} \mathbf{P} f\right\|_{\infty} \leq C(\operatorname{Re} t)^{-1 / 2}\|f\|_{\infty}
$$

for all $f \in L^{\infty}\left(\mathbf{R}^{n}\right)$ and $t \in \mathbf{C}$ with $\operatorname{Re} t>0$.

Proof. It suffices to prove that

$$
\left\|\partial_{k} e^{t \Delta} R_{i} R_{j} f\right\|_{\infty} \leq C(\operatorname{Re} t)^{-1 / 2}\|f\|_{\infty}, \quad(1 \leq i, j, k \leq n)
$$


where $R_{j}=\partial_{j}(-\Delta)^{-1 / 2}$ is the Riesz operator and $\partial_{k}=\partial / \partial x_{k}$. Since

$$
R_{i} R_{j}=\partial_{i} \partial_{j}(-\Delta)^{-1}=\partial_{i} \partial_{j} \int_{0}^{\infty} e^{s \Delta} d s
$$

we observe that

$$
\partial_{k} e^{t \Delta} R_{i} R_{j} f=e^{t \Delta} \int_{\operatorname{Re} t}^{\infty} \partial_{k} \partial_{i} \partial_{j} e^{s \Delta} d s f+\partial_{k} \partial_{i} \partial_{j} e^{t \Delta} \int_{0}^{\operatorname{Re} t} e^{s \Delta} d s f .
$$

Since

$$
\left\|\partial_{k} \partial_{i} \partial_{j} e^{t \Delta} f\right\|_{\infty} \leq C_{1}(\operatorname{Re} t)^{-3 / 2}\|f\|_{\infty}, \quad\left\|e^{t \Delta} f\right\|_{\infty} \leq\|f\|_{\infty} \text { for Re } t>0
$$

with some $C_{1}$ independent of $t$ and $f \in L^{\infty}\left(\mathbf{R}^{n}\right)$, we have

$$
\begin{aligned}
\left\|\partial_{k} e^{t \Delta} R_{i} R_{j} f\right\| & \leq C_{1} \int_{\operatorname{Re} t}^{\infty} s^{-3 / 2} d s\|f\|_{\infty}+C_{1}(\operatorname{Re} t)^{-3 / 2}(\operatorname{Re} t)\|f\|_{\infty} \\
& \leq 3 C_{1}(\operatorname{Re} t)^{-1 / 2}\|f\|_{\infty} .
\end{aligned}
$$

Remark. Lemma A for $t>0$ is proved in [10] by using a Hardy space estimate of the Gauss kernel found by Carpio [6]. A direct proof estimating integral kernels is found by Shibata and Shimizu [21]. The proof above is very short and elementary.

\section{References}

[1] N. I. Akhiezer and I. M. Glazman, Theory of linear operators in Hilbert space. F. Unger Pub. Co., New York (1961).

[2] L. Ahlfors, Complex analysis. An introduction to the theory of analytic functions of one complex variable. Third edition. International Series in Pure and Applied Mathematics. McGraw-Hill Book Co., New York (1978).

[3] M. Berger, Nonlinearity and functional analysis. Academic Press, New YorkLondon, (1977).

[4] A. Babin, A. Mahalov and B. Nicolaenko, Global regularity of the 3D Rotating Navier-Stokes Equations for resonant domains. Indiana University Mathematics Journal, 48 (1999), 1133-1176.

[5] A. Babin, A. Mahalov and B. Nicolaenko, 3D Navier-Stokes and Euler equations with initial data characterized by uniformly large vorticity. Indiana University Mathematics Journal, 50 (2001), 1-35. 
[6] A. Carpio, Large-time behaviour in incompressible Navier-Stokes equations. SIAM J. Math. Anal., 27 (1996), 449-475.

[7] C. Corduneanu, Almost Periodic Functions, Interscience Publishers, New York (1968).

[8] C. Foias and R. Temam, Some analytic and geometric properties of the solutions of the evolution Navier-Stokes equations. J. Math. Pure Appl., 58 (1979), 339366.

[9] Y. Giga, Time and spatial analyticity of solutions of the Navier-Stokes equations, Comm. in Partial Differential Equations, 8 (1983), 929-948.

[10] Y. Giga, K. Inui and S. Matsui, On the Cauchy problem for the Navier-Stokes equations with nondecaying initial data. Advances in fluid dynamics, 27-68, Quad. Mat., 4, Dept. Math., Seconda Univ. Napoli, Caserta, (1999).

[11] Y. Giga, K. Inui, A. Mahalov and S. Matsui, Navier-Stokes equations in a rotating frame in $R^{3}$ with initial data nondenessing at infinity. Hokkaido Math. J., 35 (2006), 321-364.

[12] Y. Giga, K. Inui, A. Mahalov and S. Matsui, Uniform local solvabilty for the Navier-Stokes equations with the Coriolis force. Methods Appl. Anal., 12 (2005), 381-393.

[13] Y. Giga, K. Inui and A. Mahalov and J. Saal, Global solvability of the NavierStokes equations in spaces based on sum-closed frequency sets. Adv. Diff. Eq., 12 (2007), 721-736.

[14] Y. Giga, A. Mahalov and B. Nicolaenko, The Cauchy problem for the NavierStokes equations with spatially almost periodic initial data. Hokkaido Univ. Preprint Series in Math., \#683 (2004).

[15] M. Hieber and O. Sawada, The Navier-Stokes equations in $\mathbb{R}^{n}$ with linearly growing initial data. Arch. Rational. Mech. Anal., 175 (2005), 269-285.

[16] G. Iooss, Sur la deuxieme bifucation d'une solution stationaire de systems du type Navier-Stokes equations. Arch. Rational Mech. Anal., 64 (1977), 339-369.

[17] A. Mahalov and B. Nicolaenko, Global regularity of the 3D Navier-Stokes Equations with weakly aligned large initial vorticity. Russian Math. Surveys, 58 (350), (2003), 287-318.

[18] A. Mahalov, B. Nicolaenko, C. Bardos and F. Golse, Regularity of Euler equations for a class of three-dimensional initial data. Special Volume on 'Trends in Partial Differential Equations of Mathematical Physics', Nonlinear Analysis Series, Birkhauser-Verlag, 61 (2004), 161-185.

[19] K. Masuda, On the analyticity and the unique continuation theorem for solutions of the Navier-Stokes equation. Proc. Japan Acad., 43 (1967), 827-832.

[20] O. Sawada, The Navier-Stokes flow with linearly growing initial velocity in the whole space. Bol. Soc. Parana. Mat., (3) 22, (2004), 75-96. 
[21] Y. Shibata and S. Shimizu, A decay property of the Fourier transform and its application to the Stokes problem. J. Math. Fluid Mech., 3 (2001), 213-230.

[22] K. Yosida, Functional analysis. Sixth edition. Springer-Verlag, Berlin-New York, (1980). 